\title{
Erratum to: Assessing risk of osteoporotic fractures in primary care: development and validation of the FRA-HS algorithm
}

\author{
Francesco Lapi $^{1}$ - Elisa Bianchini ${ }^{1} \cdot$ Raffaella Michieli $^{2} \cdot$ Alessandro Pasqua $^{1}$. \\ Iacopo Cricelli ${ }^{1}$ - Giampiero Mazzaglia ${ }^{1}$ - Bruno Frediani ${ }^{3}$. \\ Daniel Prieto-Alhambra $^{4,5} \cdot$ Maria Luisa Brandi ${ }^{6}$ Claudio Cricelli ${ }^{2}$
}

Published online: 13 March 2017

(C) Springer Science+Business Media New York 2017

\section{Erratum to: Calcif Tissue Int \\ DOI 10.1007/s00223-016-0230-7}

The original version of this article contained a mistake whereby the authors' surnames preceded their first names.
The correct order of the authors names is given in this erratum.

The online version of the original article can be found under doi:10.1007/s00223-016-0230-7.

Francesco Lapi

francesco@simg.it

1 Health Search, Italian College of General Practitioners and Primary Care, Florence, Italy

2 Italian College of General Practitioners and Primary Care, Florence, Italy

3 Department of Rheumatology, Policlinico Le Scotte, University of Siena, Siena, Italy

4 Oxford NIHR Musculoskeletal Biomedical Research Unit, Nuffield Department of Orthopaedics, Rheumatology and Musculoskeletal Sciences, University of Oxford, Oxford, UK

5 GREMPAL Research Group, Institut Universitari d'Investigació en Atenció Primària Jordi Gol (IDIAP Jordi Gol), Universitat Autònoma de Barcelona, Barcelona, Spain

6 Metabolic Bone Diseases Unit, Department of Surgery and Translational Medicine, University of Florence, Florence, Italy 